\title{
On the Number of Rational
}

Points of Bounded Height on

Smooth Bilinear Hypersurfaces in

\section{Biprojective Space}

\section{Journal Article}

Author(s):

Robbiani, Marcello

Publication date:

2001

Permanent link:

https://doi.org/10.3929/ethz-b-000422860

Rights / license:

In Copyright - Non-Commercial Use Permitted

Originally published in:

Journal of the London Mathematical Society 63(1), https://doi.org/10.1112/S0024610700001617 


\title{
ON THE NUMBER OF RATIONAL POINTS OF BOUNDED HEIGHT ON SMOOTH BILINEAR HYPERSURFACES IN BIPROJECTIVE SPACE
}

\author{
MARCELLO ROBBIANI
}

\begin{abstract}
Asymptotic formulae for the number of rational points of bounded height on flag varieties have earlier been established. In the paper these asymptotic formulae are recovered by a new method for varieties in biprojective space defined over $\mathbb{Q}$ that are isomorphic to the flag variety of lines in hyperplanes.

The result is obtained by an application of Heath-Brown's new form of the circle method. It serves as a pointer to the investigation of rational points of bounded height on varieties in multiprojective space.
\end{abstract}

\section{Introduction}

Let $k$ be a number field. In the last few years a great deal of effort has been exerted to establish some asymptotic formulae for the number of $k$-rational points of bounded height on Fano varieties $X$. One expects results of the following form.

Suppose that the $k$-rational points are dense in $X$ and that there exists a Zariskiopen subset $U$ of $X$ defined over $k$, in which the $k$-rational points are in some precise sense 'homogeneous' and their density 'minimal'. Let $H$ be one of the multiplicative anticanonical heights on $X$ and let $\rho$ be the rank of the Picard group Pic $X$. Then, as $B$ tends to infinity, the integer

satisfies

$$
N(U, B)=\operatorname{card}\{P \in U(k) \mid H(P)<B\}
$$

$$
N(U, B) \sim C B(\log (B))^{\rho-1},
$$

where $C$ is some constant that depends only on $X$ and $k$.

Asymptotic formulae of this type have been established for certain classes of Fano varieties, for example in $[\mathbf{1}-\mathbf{3}, \mathbf{6}, \mathbf{1 2}, \mathbf{1 5}, \mathbf{1 6}, \mathbf{2 0}]$. These results are in agreement with general interpretations by Batyrev, Manin and Peyre, which state a deep relationship between geometry and arithmetic on Fano varieties. For precise statements we refer for example to $[\mathbf{1}, \mathbf{6}, \mathbf{1 2}]$.

For a summary of the research in this area we may also refer to [11, 'Anticanonical varieties and rational points'].

\section{On the method of Schanuel, Peyre and Salberger}

A method that has been developed in $[\mathbf{1 2}, \mathbf{1 6}, \mathbf{1 7}]$ to tackle this type of problem is now presented.

Received 15 October 1998; final revision 18 April 2000.

2000 Mathematics Subject Classification 11G35.

J. London Math. Soc. (2) 63 (2001) 33-51. (C) London Mathematical Society 2001. 
Let $\mathcal{O}$ be the ring of integers of $k$. One proves, under some additional conditions on $X$, that there exist a finite set of places $S$ of $k, \mathcal{O}_{S}$-models $\tilde{X}$ of $X$ and $\tilde{R}$ of the Néron-Severi-torus of $X$ (the dual of the Picard-group-scheme), and a finite family of $\mathcal{O}_{S}$-schemes $\mathscr{T}^{\alpha}$, such that

$$
p^{\alpha}: \mathscr{T}^{\alpha} \longrightarrow \tilde{X}
$$

are torsors (principal homogeneous spaces) over $\tilde{X}$ under $\tilde{R}$, and such that as a disjoint union

$$
\bigcup_{\alpha} p^{\alpha} \mathscr{T}^{\alpha}\left(\mathcal{O}_{S}\right)=\tilde{X}\left(\mathcal{O}_{S}\right)
$$

As $X$ is projective one also has

$$
X(k)=\tilde{X}\left(\mathcal{O}_{S}\right) .
$$

Thus, after choosing sets $\mathfrak{D}^{\alpha}$ of representatives for the action of $\tilde{R}\left(\mathcal{O}_{S}\right)$, one obtains a one-to-one parametrisation of the $k$-rational points on $X$ by the $\mathcal{O}_{S}$-points in

$$
\bigcup_{\alpha} \mathfrak{D}^{\alpha} \cap \mathscr{T}^{\alpha}\left(\mathcal{O}_{S}\right)
$$

For toric varieties each $\mathscr{T}^{\alpha}$ is the complement in some $\mathbb{A}_{\mathscr{S}_{S}}^{n}$ of a finite union of subvarieties of at least codimension 2. Hence, the initial problem reduces to that of counting points in $\mathbb{A}^{n}\left(\mathcal{O}_{S}\right)$ that satisfy some primitiveness conditions and various inequalities. Such lattice point problems are classical and can be solved by methods from algebraic number theory and the geometry of numbers.

The next natural class of varieties to be considered is the class of smooth hypersurfaces $X$ in a toric Fano variety $Y$. In this case, one can try to attack the problem by the following programme.

If $Y$ is, say, of dimension greater than or equal to 3, then Pic $X$ and Pic $Y$ are, by a well known theorem of Grothendieck and Lefschetz (see for example [7, Chapter IV, Theorem 3.1 and Corollary 3.3]), isomorphic, and one can find torsors $\mathscr{T}_{X}^{\alpha}$ over $X$ with the above properties by restricting similar torsors

$$
p^{\alpha}: \mathscr{T}_{Y}^{\alpha} \longrightarrow Y \text {. }
$$

Therefore the $\mathscr{T}_{X}^{\alpha}$ are open subsets of hypersurfaces in affine spaces. Thus choosing appropriate $\mathcal{O}_{S}$-models one can parametrise the $k$-rational points on $X$ by the $\mathcal{O}_{S}$-points on affine hypersurfaces that satisfy some primitiveness conditions and various inequalities. One hopes to solve this last problem, at least for certain classes of varieties, by the circle method.

This hope is justified by the fact that, when $Y$ is the projective space, various results obtained by the circle method, for example in $[\mathbf{4}, \mathbf{8}, \mathbf{9}, \mathbf{1 8}]$, fit into this programme. For a discussion in detail we may refer to $[\mathbf{6}, \mathbf{1 2}]$.

\section{On bilinear hypersurfaces in biprojective space}

Let $n \geqslant 2$, and let $X$ be a hypersurface in $\mathbb{P}_{\mathbb{Q}}^{n} \times \mathbb{P}_{\mathbb{Q}}^{n}$ defined by an equation

$$
F(\mathbf{x} \times \mathbf{y})=\sum_{i=0}^{n} \sum_{j=0}^{n} m_{i j} x_{i} y_{j}=0,
$$

where $\mathbf{x} \times \mathbf{y}$ or $\left(x_{0}: \ldots: x_{n}\right) \times\left(y_{0}: \ldots: y_{n}\right)$ are bihomogeneous coordinates, and $\left[m_{i j}\right]$ is a matrix with entries in $\mathbb{Q}$. Note that $X$ is smooth if and only if $\left[m_{i j}\right]$ is regular. The dimension of $X$ is $2 n-1$. 
Let $\tilde{Y}$ be the subscheme of $\mathbb{P}_{\mathbb{Z}}^{n} \times \mathbb{P}_{\mathbb{Z}}^{n}$, defined by $\mathbf{x} \cdot \mathbf{y}=0$. As $\left[m_{i j}\right]$ is regular, one has

$$
X \cong Y=\tilde{Y} \otimes_{\mathbb{Z}} \mathbb{Q} \text {. }
$$

The condition $\mathbf{x} \cdot \mathbf{y}=0$ may be seen as an incidence relation. In fact $X$ is isomorphic to the flag variety of lines in hyperplanes.

Recall that $\mathbb{P}_{\mathbb{Q}}^{n} \times \mathbb{P}_{\mathbb{Q}}^{n}$ is a smooth toric Fano variety, that is a variety with ample anticanonical class. By the adjunction formula the anticanonical sheaf of $X$ can be obtained by restriction from $\mathbb{P}^{n} \times \mathbb{P}^{n}$ to $X$ of the sheaf $\mathcal{O}(n, n)$. It follows that $X$ is itself Fano. An embedding of $X$ into projective space can be given by a choice of a set of generators for the space of global sections $\Gamma(X, O(n, n))$, that is by a set of monomials of bidegree $(n, n)$. Such an embedding will be called anticanonical.

For any projective embedding $\phi$ of $X$ the notion of height can be defined. Let $\mathfrak{M}$ be the set of all places of $\mathbb{Q}$, and let ||$_{\wp}$ be for all $\wp \in \mathfrak{M}$ the usual $\wp$-adic norm (we write at infinity || for the usual real norm). The height $H(\mathbf{p})$ of a $\mathbb{Q}$-rational point $\mathbf{p}$ in projective space represented by homogeneous coordinates $\left(p_{0}: \ldots: p_{i}: \ldots: p_{n}\right)$ is defined by the formula

$$
H(\mathbf{p})=\prod_{\mathfrak{\wp} \in \mathfrak{M}} \max _{i}\left|p_{i}\right|_{\wp} .
$$

This height is by the product formula

$$
\prod_{\wp \in M}|q|_{\wp}=1
$$

for $q \in \mathbb{Q}^{*}$ independent on the choice of the representative coordinates. In particular if $\mathbf{p}$ is represented by relatively prime integral coordinates one has

$$
H(\mathbf{p})=\max _{i}\left|p_{i}\right| .
$$

The height $H(\mathbf{x} \times \mathbf{y})$ of a point $\mathbf{x} \times \mathbf{y}$ of $X(\mathbb{Q})$ attached to an embedding $\phi$ is defined by the formula

$$
H_{\phi}(\mathbf{x} \times \mathbf{y})=H(\phi(\mathbf{x} \times \mathbf{y})) .
$$

As outlined in the introduction one is interested in the asymptotical behaviour of counting functions

$$
N(X, \phi, B)=\operatorname{card}\left\{\mathbf{x} \times \mathbf{y} \in X(\mathbb{Q}) \mid H_{\phi}(\mathbf{x} \times \mathbf{y}) \leqslant B\right\} .
$$

Asymptotic formulae for counting functions of rational points on flag varieties have been established in $[\mathbf{6}, \mathbf{2 0}]$. The purpose of this paper is to give an alternative proof of the following theorem.

THEOREM 1. Assume that $n \geqslant 3$, and let $\phi$ be an embedding attached to the sheaf $O(1,1)$. There exists a positive constant $C$ such that for $B$ tending to infinity

$$
N(X, \phi, B) \sim C B^{n} \log (B) .
$$

$C$ depends on the embedding, however, the order of magnitude does not. In fact, by a theorem of Weil (see for example [10]), for any pair of embeddings $\phi$ and $\phi^{\prime}$ attached to the sheafs $O(1,1)$ and $O(n, n)$, respectively, there exists a positive constant $C$, such that for any point $\mathbf{x} \times \mathbf{y}$ in $X(\mathbb{Q})$ one has

$$
C^{-1} H_{\phi}(\mathbf{x} \times \mathbf{y})^{n} \leqslant H_{\phi^{\prime}}(\mathbf{x} \times \mathbf{y}) \leqslant C H_{\phi}(\mathbf{x} \times \mathbf{y})^{n} .
$$

It follows in particular, as the rank of Pic $X$ is 2, that this result is in accordance with the general philosophy outlined in the introduction. 
By similar arguments, one sees that for any embedding $\phi$ attached to $O(1,1)$ the behaviour of $N(X, \phi, B)$ for $B$ tending to infinity is similar to the one of $N(Y, \psi, B)$, where $\psi$ is the Segre embedding that maps a point $\mathbf{x} \times \mathbf{y}$ to

$$
\left(x_{0} y_{0}: \ldots: x_{i} y_{j}: \ldots: x_{n} y_{n}\right) \text {. }
$$

It suffices therefore to prove Theorem 1 for $N(Y, \psi, B)$.

The method of Schanuel, Peyre and Salberger reduces in this case to the fact that each point in $Y(\mathbb{Q})$ can be represented uniquely by an integral point in its affine cone whose coordinates are relatively prime and contained in a fundamental domain for the four possible choices of signs. Indeed, let $\mathscr{T}$ be the regular subscheme of $\mathbb{A}_{\mathbb{Z}}^{n+1} \times \mathbb{A}_{\mathbb{Z}}^{n+1}$ associated to the affine cone of $Y$. One can show that $\mathscr{T}$ is a torsor over $\tilde{Y}$ under $\mathbb{G}_{m}^{2}$, the Néron-Severi-torus of $\tilde{Y}$. Hence, each point in $\tilde{Y}(\mathbb{Z})$ is (up to the action of $\left.\mathbb{G}_{m}^{2}(\mathbb{Z})\right)$ in bijection with a point in $\mathscr{T}(\mathbb{Z})$.

Define

$$
H(\mathbf{x} \times \mathbf{y})=\max _{i, j}\left|x_{i} y_{j}\right|
$$

where $\mathbf{x} \times \mathbf{y}$ or $\left(x_{0}, \ldots, x_{n}\right) \times\left(y_{0}, \ldots, y_{n}\right)$ are now affine coordinates. Then for integral points given by relatively prime coordinates one has (carrying on with this precarious notation) the identity

$$
H(\mathbf{x} \times \mathbf{y})=H_{\psi}(\mathbf{x} \times \mathbf{y}) .
$$

Now, to determine the asymptotical behaviour of $N(Y, \psi, B)$, one will proceed as follows. At a first stage one forgets about the primitiveness condition and establishes an asymptotic formula for $N(B)$, the cardinality of the set

$$
\mathscr{S}(B)=\left\{\mathbf{x} \times \mathbf{y} \in \mathbb{Z}^{n+1} \times \mathbb{Z}^{n+1} \mid x_{\max }>0, y_{\max }>0, \mathbf{x} \cdot \mathbf{y}=0, H(\mathbf{x} \times \mathbf{y}) \leqslant B\right\},
$$

where the index ' $m a x$ ' indicates the coordinate whose absolute value is maximal. At a second stage, one takes into account of the primality conditions by a sieveargument, the so-called Möbius-inversion, which is particularly simple in this case.

\section{On the method of Heath-Brown}

We begin by defining

and

$$
\omega_{0}(x)= \begin{cases}\exp \left(-\left(1-x^{2}\right)^{-1}\right), & |x|<1 \\ 0, & |x| \geqslant 1\end{cases}
$$

as well as

$$
c_{0}=\int_{-\infty}^{\infty} \omega_{0}(x) d x
$$

and

$$
\omega(x)=4 c_{0}^{-1} \omega_{0}(4 x-3)
$$

$$
h(x, y)=\sum_{j} \frac{1}{x j}\{\omega(x j)-\omega(|y| / x j)\} .
$$

REMARK 2. $h(x, y)$ is an infinitely differentiable real function defined on $(0, \infty) \times \mathbb{R}$, which is non-zero only for

and which satisfies for all $y$

$$
x \leqslant \max (1,2|y|)
$$

$$
|h(x, y)| \ll \frac{1}{x}
$$


The following result is due to Duke, Friedlander and Iwaniec [5].

THEOREM 3. For any integer $n$ let

$$
\delta_{n}= \begin{cases}1, & n=0, \\ 0, & n \neq 0 .\end{cases}
$$

Then for any $Q>1$ there is a positive constant $c_{Q}$, which satisfies

for any $N>0$, such that

$$
c_{Q}=1+O_{N}\left(Q^{-N}\right)
$$

$$
\delta_{n}=c_{Q} Q^{-2} \sum_{q=1}^{\infty} \sum_{d(q)}^{*} e_{q}(d n) h\left(\frac{q}{Q}, \frac{n}{Q^{2}}\right) .
$$

Here the notation $e(x)$ for $\exp (2 \pi i x), e_{q}(x)$ for $e(x / q)$, and $d(q)$ for $d \bmod q$, has been introduced. Moreover $\sum^{*}$ indicates a summation for residues $d$ with $(d, q)=1$.

Let $F(\mathbf{x})$ be a polynomial in $n$ variables with coefficients in $\mathbb{Z}$, and let $w(\mathbf{x})$ be a bounded function well-defined on the integral points of a compact subset of $\mathbb{R}^{n}$. The aim is to estimate

$$
N(F, w)=\sum w(\mathbf{x}),
$$

the sum being taken over all $\mathbf{x} \in \mathbb{Z}^{n}$ for which $F(\mathbf{x})=0$.

Observe that

$$
N(F, w)=\sum w(\mathbf{x}) \delta_{F(\mathbf{x})} .
$$

Hence Theorem 3 leads immediately to the following corollary.

Corollary 4. For any constant $Q>1$

with

$$
N(F, w)=\sum_{\mathbf{x}} c_{Q} Q^{-2} \sum_{q=1}^{\infty} \sum_{d(q)} * w(\mathbf{x}) e_{q}(d F(\mathbf{x})) h\left(\frac{q}{Q}, \frac{F(\mathbf{x})}{Q^{2}}\right),
$$

for any $N>0$.

$$
c_{Q}=1+O_{N}\left(Q^{-N}\right)
$$

The starting point of the method of Heath-Brown is the following result, which can be derived from Corollary 4 .

THEOREM 5. If, moreover, $w$ is a 'smooth weight function', that is an infinitely differentiable function with compact support, then

where

$$
N(F, w)=\sum_{\mathbf{c} \in \mathbb{Z}^{n}} \sum_{q=1}^{\infty} q^{-n} S_{q}(\mathbf{c}) I_{q}(\mathbf{c}),
$$

and

$$
\left.S_{q}(\mathbf{c})=\sum_{d(q)} * \sum_{\mathbf{b}(q)} e_{q}(d F(\mathbf{b})+\mathbf{c} \cdot \mathbf{b})\right),
$$

$$
I_{q}(\mathbf{c})=\int_{\mathbb{R}^{n}} c_{Q} Q^{-2} w(\mathbf{x}) h\left(\frac{q}{Q}, \frac{F(\mathbf{x})}{Q^{2}}\right) e_{q}(-\mathbf{c} \cdot \mathbf{x}) d \mathbf{x} .
$$

This is [9, Theorem 2]. 
REMARK 6. For a generalisation along the lines of [13] of the classical circle method and its application to hypersurfaces of the former type we refer to [14].

\section{On smooth weights}

Let $0<\varepsilon<1 / 4 n$. Define the weights

$$
\underline{\omega}_{\varepsilon}(x)=c_{0}^{-1} \varepsilon^{-1} \int_{-\infty}^{x-\varepsilon} \omega_{0}\left(\varepsilon^{-1} y\right) d y,
$$

and $\bar{\omega}_{\varepsilon}(x)$ by replacing ' $x-\varepsilon$ ' by ' $x+\varepsilon$ '.

Assume now that, say,

$$
x_{0}=\max _{i}\left|x_{i}\right|, \quad y_{0}=\max _{i}\left|y_{i}\right| .
$$

This is case A. Case B, in which the maximal indices differ, can be treated similarly, and is in fact slightly simpler. Some details are given in Section 6 and in Section 7.

Set

$$
\mathbf{x}=\left(x_{1}, \ldots, x_{n}\right), \quad \mathbf{y}=\left(y_{1}, \ldots, y_{n}\right) .
$$

Define $\underline{w}_{\varepsilon, B,(0,0)}=\underline{w}_{\varepsilon, B}\left(x_{0}, y_{0}, \mathbf{x}, \mathbf{y}\right)$ as the product of

and

$$
\underline{w}_{\varepsilon, B}\left(x_{0}, y_{0}\right)=\underline{\omega}_{\varepsilon}\left(x_{0}-1\right) \underline{\omega}_{\varepsilon}\left(B-x_{0} y_{0}\right) \underline{\omega}_{\varepsilon}\left(y_{0}-\sqrt{B}\right),
$$

$$
\underline{w}_{\varepsilon}\left(x_{0}, \mathbf{x}\right)=\prod_{i \neq 0} \underline{\omega}_{\varepsilon}\left(1-\left|x_{i}\right| / x_{0}\right) w_{e}\left(x_{0}, \mathbf{x}\right)
$$

with

as well as

$$
w_{e}\left(x_{0}, \mathbf{x}\right)=1-\left(\prod_{i \neq 0} \bar{\omega}_{\varepsilon}\left(1 / 2 n-\left|x_{i}\right| / x_{0}\right)\right),
$$

$$
\underline{w}_{\varepsilon}\left(y_{0}, \mathbf{y}\right)=\prod_{i \neq 0} \underline{\omega}_{\varepsilon}\left(1-\left|y_{i}\right| / y_{0}\right) .
$$

Define $\underline{w}_{\varepsilon, B,(\mathbf{0}, \mathbf{0})}=\bar{w}_{\varepsilon, B}\left(x_{0}, y_{0}, \mathbf{x}, \mathbf{y}\right)$ in a similar way.

This choice is justified on the one hand by the fact that by [9, Theorem 5] the number of points whose coordinates are bounded by $\sqrt{B}$ is $O\left(B^{n}\right)$. On the other hand in Section 7 it will be shown that there exists a positive constant $C_{0,0}$ and a function $B(\varepsilon)$ such that for $B \geqslant B(\varepsilon)$

where

$$
\left|N\left(F, \underline{w}_{\varepsilon, B}\left(x_{0}, y_{0}, \mathbf{x}, \mathbf{y}\right)\right)-C_{0,0} B^{n} \log (B)\right| \leqslant O(\varepsilon) B^{n} \log (B),
$$

$$
F\left(x_{0}, y_{0}, \mathbf{x}, \mathbf{y}\right)=x_{0} y_{0}+\mathbf{x} \cdot \mathbf{y} .
$$

It will also be shown that a similar inequality with the same constant $C_{0,0}$ holds if $\underline{w}$ is replaced by $\bar{w}$. Similar results, with constants $C_{i, j}$, hold for all other choices of pairs of indexes.

Hence, one concludes for $B$ tending to infinity that

$$
N(B) \sim 2 \sum_{i, j} N\left(F, w_{\varepsilon, B,(i, j)} \sim 2 \sum_{i, j} C_{i, j} B^{n} \log (B),\right.
$$

where it makes no difference if $w$ is underlined or overlined. Note that the asymmetries of the weights are corrected by the factor 2 . 
The identity

implies the extra condition

$$
x_{0} y_{0}+\mathbf{x} \cdot \mathbf{y}=0
$$

$$
\left|x_{i}\right| \geqslant \frac{x_{0}}{n}
$$

for at least one index $i \neq 0$. Thus the extra factor $w_{e}$ has no influence on the counting problem. As will become clear in Section 7, its role is purely technical.

REMARK 7. In case B no extra factor is needed.

Finally, one is led to evaluate $N\left(F, w_{\varepsilon, B}\left(x_{0}, y_{0}, \mathbf{x}, \mathbf{y}\right)\right)$. Drop all the indices and subscripts and write this cardinal as

$$
\sum_{x_{0}, y_{0} \mathbf{x}, \mathbf{y}} w\left(x_{0}, y_{0}, \mathbf{x}, \mathbf{y}\right) \delta_{F\left(x_{0}, y_{0}, \mathbf{x}, \mathbf{y}\right)} .
$$

Split the inner sum into

$$
\sum_{x_{0}, y_{0}} w\left(x_{0}, y_{0}\right) \sum_{\mathbf{x}} w\left(x_{0}, \mathbf{x}\right) \sum_{\mathbf{y}} w\left(y_{0}, \mathbf{y}\right) \delta_{F\left(x_{0}, y_{0}, \mathbf{x}, \mathbf{y}\right)},
$$

and apply to the innermost sum Corollary 4 with

$$
Q=\sqrt{x_{0} y_{0}} \text {. }
$$

Then Theorem 5 states that the innermost sum can be replaced by

$$
\sum_{\mathbf{c} \in \mathbb{Z}^{n}} \sum_{q=1}^{\infty} q^{-n} S_{q}\left(x_{0}, y_{0}, \mathbf{x}\right)(\mathbf{c}) I_{q}\left(x_{0}, y_{0}, \mathbf{x}\right)(\mathbf{c}),
$$

where $S_{q}\left(x_{0}, y_{0}, \mathbf{x}\right)(\mathbf{c})$ is equal to

$$
\sum_{d(q)} * \sum_{\mathbf{b}(q)} e_{q}\left(d F\left(x_{0}, y_{0}, \mathbf{x}, \mathbf{b}\right)+\mathbf{c} \cdot \mathbf{b}\right),
$$

and $I_{q}\left(x_{0}, y_{0}, \mathbf{x}\right)(\mathbf{c})$ is up to a factor $c\left(x_{0}, y_{0}\right)$ equal to

$$
\int_{\mathbb{R}^{n}} \frac{w\left(y_{0}, \mathbf{y}\right)}{x_{0} y_{0}} h\left(\frac{q}{\sqrt{x_{0} y_{0}}}, \frac{x_{0} y_{0}+\mathbf{x} \cdot \mathbf{y}}{x_{0} y_{0}}\right) e_{q}(-\mathbf{c} \cdot \mathbf{y}) d \mathbf{y} .
$$

As on $\operatorname{supp} w\left(x_{0}, y_{0}, \mathbf{x}, \mathbf{y}\right)$

$$
\left|\frac{x_{0} y_{0}+\mathbf{x} \cdot \mathbf{y}}{x_{0} y_{0}}\right| \ll n+1,
$$

by Remark 2 these integrals are non-zero only if

$$
q \ll \sqrt{x_{0} y_{0}} .
$$

Therefore this condition will be set through this paper as a general assumption.

Note that despite having defined weights on all $x_{i}$ and $y_{j}$ Theorem 5 is in fact applied only to $\mathbf{y}$. Hence the preceding step may be viewed as an application of the method of Heath-Brown to an inhomogeneous linear equation.

\section{On the error term}

This section is devoted to the proof of the following proposition. 
Proposition 8. The expression

$$
\left|\sum_{x_{0}, y_{0}} w\left(x_{0}, y_{0}\right) \sum_{\mathbf{x} \in \mathbb{Z}^{n}} w\left(x_{0}, \mathbf{x}\right) \sum_{\substack{\mathbf{c} \in \mathbb{Z}^{n} \\ \mathbf{c} \neq 0}} \sum_{q \ll \sqrt{x_{0} y_{0}}} q^{-n} S_{q}\left(x_{0}, y_{0}, \mathbf{x}\right)(\mathbf{c}) I_{q}\left(x_{0}, y_{0}, \mathbf{x}\right)(\mathbf{c})\right|
$$

is $O_{\varepsilon}\left(B^{n}\right)$.

To handle $I_{q}\left(x_{0}, y_{0}, \mathbf{x}\right)(\mathbf{c})$ write

$$
\mathbf{y}=y_{0} \mathbf{v}
$$

Then $I_{q}\left(x_{0}, y_{0}, \mathbf{x}\right)$ (c) transforms up to the factor $x_{0}^{-1} y_{0}^{n-1}$ (and the negligible factor $\left.c\left(x_{0}, y_{0}\right)\right)$ into

$$
\int_{\mathbb{R}^{n}} w(\mathbf{v}) h\left(\frac{q}{\sqrt{x_{0} y_{0}}}, 1+\mathbf{x} / x_{0} \cdot \mathbf{v}\right) e_{q}\left(\mathbf{c} \cdot y_{0} \mathbf{v}\right) d \mathbf{v}
$$

where $w(\mathbf{v})$ is the corresponding transformed weight.

Set

$$
\omega_{1}(s)=\omega_{0}\left(\frac{s}{2 n}\right)
$$

Then, as in [9, Section 7], one may use the Fourier transform to write

$$
\omega_{1}\left(1+\mathbf{x} / x_{0} \cdot \mathbf{v}\right) h\left(\frac{q}{\sqrt{x_{0} y_{0}}}, 1+\mathbf{x} / x_{0} \cdot \mathbf{v}\right)=\int_{-\infty}^{\infty} p(t) e\left(t\left(1+\mathbf{x} / x_{0} \cdot \mathbf{v}\right)\right) d t
$$

where

$$
p(t)=\int_{-\infty}^{\infty} h\left(\frac{q}{\sqrt{x_{0} y_{0}}}, s\right) \omega_{1}(s) e(-t s) d s .
$$

On $\operatorname{supp} w(\mathbf{v})$ (and for the $x_{0}$ and $\mathbf{x}$ one is interested in) the expression

$$
\left|1+\mathbf{x} / x_{0} \cdot \mathbf{v}\right|
$$

is bounded by $2 n$, and therefore the expression

$$
\omega_{1}\left(1+\mathbf{x} / x_{0} \cdot \mathbf{v}\right)
$$

is strictly greater than some positive constant. Hence integral (3) may be expressed as

where

$$
\int_{-\infty}^{\infty} \int_{\mathbb{R}^{n}} p(t) \psi(\mathbf{v}) e(\phi(\mathbf{v}, t)) d \mathbf{v} d t
$$

and

$$
\psi(\mathbf{v})=\frac{w(\mathbf{v})}{\omega_{1}\left(1+\mathbf{x} / x_{0} \cdot \mathbf{v}\right)}
$$

$$
\phi(\mathbf{v}, t)=t\left(1+\mathbf{x} / x_{0} \cdot \mathbf{v}\right)-\frac{\mathbf{c}}{q} \cdot y_{0} \mathbf{v} .
$$

One needs now several results on oscillatory integrals. One could invoke [19, Chapter VIII] or follow [9, Section 6, Section 7]. In what follows the second alternative has been chosen. 
LemMa 9. For all $\mathbf{c} \neq \mathbf{0}$, for all sufficiently small $\varepsilon>0$, and for all positive integers $N$ one has

$$
\left|\int_{|t| \leqslant(1 / 2 n)(\mathbf{c} \mid / q) y_{0}} \int_{\mathbb{R}^{n}} p(t) \psi(\mathbf{v}) e(\phi(\mathbf{v}, t)) d \mathbf{v} d t\right| \ll_{\varepsilon, N}|\mathbf{c}|^{-2 N} x_{0}^{N} y_{0}^{-N} .
$$

Set, say,

$$
\left|c_{1}\right|=\max _{i}\left|c_{i}\right|
$$

To prove Lemma 9 use now the fact that inside supp $\psi$ and for

$$
|t| \leqslant \frac{1}{2 n} \frac{|\mathbf{c}|}{q} y_{0}
$$

the partial derivative of $\phi$ with respect to $v_{1}$ satisfies

$$
\left|t x_{1} / x_{0}-y_{0} \frac{c_{1}}{q}\right| \geqslant \frac{1}{3} y_{0} \frac{\left|c_{1}\right|}{q}
$$

recall that $\left|x_{1}\right| / x_{0} \leqslant 1+\varepsilon$.

On repeated integration by parts and estimating $q$ by (2) Lemma 9 follows from

$$
\begin{aligned}
\int_{\mathbb{R}} \psi(\mathbf{v}) e(\phi(\mathbf{v})) d v_{1} & =\int_{\mathbb{R}} \psi(\mathbf{v}) D^{2 N}(e(\phi(\mathbf{v}))) d v_{1} \\
& =\int_{\mathbb{R}}\left(D^{t}\right)^{2 N}(\psi(\mathbf{v})) e(\phi(\mathbf{v})) d v_{1},
\end{aligned}
$$

where $D$ stands for the differential operator

and $D^{t}$ for its transpose

$$
D f\left(v_{1}\right)=\left(i \phi^{\prime}\left(v_{1}\right)\right)^{-1} f^{\prime}\left(v_{1}\right)
$$

$$
D^{t} f\left(v_{1}\right)=-\left(\frac{f\left(v_{1}\right)}{i \phi^{\prime}\left(v_{1}\right)}\right)^{\prime}
$$

and from the fact that Remark 2 yields

$$
\int_{\mathbb{R}} p(t) d t \ll 1
$$

Lemma 10. For all sufficiently small $\varepsilon>0$, for all $\mathbf{c}$ with

$$
|\mathbf{c}|>\left(c_{0} \frac{\sqrt{x_{0} y_{0}}}{q}\right)^{\varepsilon}
$$

and for all positive integers $N>1 / \varepsilon$ one has

$$
\left|\int_{|t| \geqslant(1 / 2 n)(\mathbf{l} \mid / q) y_{0}} \int_{\mathbb{R}^{n}} p(t) \psi(\mathbf{v}) e(\phi(\mathbf{v}, t)) d \mathbf{v} d t\right| \ll_{\varepsilon, N}|\mathbf{c}|^{-N} x_{0}^{N} y_{0}^{-N} .
$$

Lemma 10 is an immediate consequence of the second statement of [9, Lemma 17] (note that the function $p$ in [9] contains an additional multiplicative factor $q / \sqrt{x_{0} y_{0}}$ ). 
Lemma 11. For all positive integers $N$ one has

$$
|p(t)| \leqslant\left(\frac{\sqrt{x_{0} y_{0}}}{q t}\right)^{N}
$$

By (2) there exists a positive constant $c_{0}$ such that

$$
q \leqslant c_{0} \sqrt{x_{0} y_{0}} .
$$

Following [9] a point $\mathbf{x} \in \mathbb{Z}^{n}$ such that $\operatorname{grad} \phi$ is small at $\mathbf{x}$ is called a 'bad point'. Precisely, $\mathbf{x}$ is said to be 'bad', if its distance from the line segment

$$
\mathbf{x}=\frac{x_{0} y_{0}}{q t} \mathbf{c}
$$

with

$$
|t| \geqslant \frac{1}{2 n} \frac{|\mathbf{c}|}{q} y_{0}
$$

is smaller than

$$
\left(c_{0} \frac{\sqrt{x_{0} y_{0}}}{q}\right)^{\varepsilon}
$$

Lemma 12. For all $|\mathbf{c}|$ with

$$
\mathbf{c} \leqslant\left(\frac{\sqrt{x_{0} y_{0}}}{q}\right)^{\varepsilon}
$$

for all positive integers $N$, and for all $\mathbf{x}$ that are not 'bad' one has

$$
\left|\int_{|t| \geqslant(1 / 2 n)(\mathbf{c} \mid / q) y_{0}} \int_{\mathbb{R}^{n}} p(t) \psi(\mathbf{v}) e(\phi(\mathbf{v}, t)) d \mathbf{v} d t\right| \ll_{\varepsilon, N}|\mathbf{c}|^{-2 N} x_{0}^{N} y_{0}^{-N} .
$$

If $\mathbf{x}$ is 'bad' then one has instead

$$
\left|\int_{|t| \geqslant(1 / 2 n)(\mathbf{l} \mid / q) y_{0}} \int_{\mathbb{R}^{n}} p(t) \psi(\mathbf{v}) e(\phi(\mathbf{v}, t)) d \mathbf{v} d t\right| \ll_{\varepsilon, N}|\mathbf{c}|^{-2 N} \frac{\sqrt{x_{0} y_{0}}}{q} x_{0}^{N} y_{0}^{-N} .
$$

The first statement in Lemma 12 is obtained on the one hand, following the proof of Lemma 9, on repeated integration by parts with respect to the variable $v_{i}$ for which $\phi^{\prime}\left(v_{i}\right)$ is maximal at $\mathbf{x}$. On the other hand one makes use of the estimate of Lemma 11 as in the proof of Lemma 10.

The second statement in Lemma 12 is weaker, as integration by parts is no longer available. It follows as Lemma 10 immediately from Lemma 11.

Putting Lemmas 9, 10 and 12 together one is led to evaluate the expressions

$$
\sum_{\mathbf{x}} w\left(x_{0}, \mathbf{x}\right) \sum_{\mathbf{c} \neq 0} \sum_{q \ll \sqrt{x_{0} y_{0}}} q^{-n} S_{q}\left(x_{0}, y_{0}, \mathbf{x}\right)(\mathbf{c})|\mathbf{c}|^{-N} x_{0}^{N-1} y_{0}^{-N+n-1}
$$

and

$$
\sum_{\mathbf{x} \text { 'bad }} w\left(x_{0}, \mathbf{x}\right) \sum_{\mathbf{c} \neq 0} \sum_{q \ll \sqrt{x_{0} y_{0}}} q^{-n-1} S_{q}\left(x_{0}, y_{0}, \mathbf{x}\right)(\mathbf{c})|\mathbf{c}|^{-N} \sqrt{x_{0} y_{0}} x_{0}^{N-1} y_{0}^{-N+n-1} .
$$

Note that in the first expression one could limit the summation to the $\mathbf{x}$ which are not 'bad'.

To tackle expression (4) one uses the following lemma. 
Lemma 13. For all $\mathbf{c} \neq \mathbf{0}$ one has

$$
\left|\sum_{\mathbf{x}} w\left(x_{0}, \mathbf{x}\right) S_{q}\left(x_{0}, y_{0}, \mathbf{x}\right)(\mathbf{c})\right| \ll q \max \left\{q^{n}, x_{0}^{n}\right\} .
$$

Indeed, write the left-hand side as

$$
\left|\sum_{d(q)}^{*} \sum_{\mathbf{x}} w\left(x_{0}, \mathbf{x}\right) \sum_{\mathbf{b}(q)} e_{q}\left(d x_{0} y_{0}+(d \mathbf{x}+\mathbf{c}) \cdot \mathbf{b}\right)\right|,
$$

and observe that by elementary properties of Gauss sums this is equal to

$$
\left|\sum_{d(q)} * e_{q}\left(d x_{0} y_{0}\right) \sum_{\mathbf{x} \equiv-d^{*} \mathbf{c}(q)} w\left(x_{0}, \mathbf{x}\right) q^{n}\right|,
$$

where $d^{*}$ is defined by $d^{*} d \equiv 1(q)$. Lemma 13 follows now from

$$
\sum_{\mathbf{x} \equiv-d^{*} \mathbf{c}(q)} w\left(x_{0}, \mathbf{x}\right) \ll \max \left\{1, \frac{x_{0}^{n}}{q^{n}}\right\} .
$$

Applying Lemma 13 to expression (4) one is finally led to evaluate

$$
\sum_{x_{0}, y_{0}} w\left(x_{0}, y_{0}\right) x_{0}^{N-1} y_{0}^{-N+n-1} \sum_{\mathbf{c} \neq \mathbf{0}}|\mathbf{c}|^{-N} \sum_{q \ll \sqrt{x_{0} y_{0}}} q^{1-n} \max \left\{q^{n}, x_{\mathbf{0}}^{n}\right\},
$$

which is $O\left(B^{n}\right)$ for an appropriate choice of $N$.

To tackle expression (5) one makes use of the observation that the maximal number of 'bad points' contained in $\operatorname{supp} w(\mathbf{x})$ is bounded by a multiple of

$$
x_{0}\left(c_{0} \frac{\sqrt{x_{0} y_{0}}}{q}\right)^{\varepsilon(n-1)} .
$$

With the trivial estimate

$$
\left|S_{q}\left(x_{0}, y_{0}, \mathbf{x}\right)(\mathbf{c})\right| \leqslant q^{n+1},
$$

one is finally led to evaluate

$$
\sum_{x_{0}, y_{0}} w\left(x_{0}, y_{0}\right) \sqrt{x_{0} y_{0}}{ }^{1+\varepsilon(n-1)} x_{0}^{N} y_{0}^{-N+n-1} \sum_{q \ll \sqrt{x_{0} y_{0}}} q^{-\varepsilon(n-1)} \sum_{\mathbf{c} \neq \mathbf{0}}|\mathbf{c}|^{-N},
$$

which is $O\left(B^{n / 2+1}\right)$ for an appropriate choice of $N$ and $\varepsilon$. This concludes the proof of Proposition 8.

REMARK 14. In case B assume that, say,

Choosing

$$
x_{1}=\max _{i}\left|x_{i}\right|, \quad y_{0}=\max _{i}\left|y_{i}\right| .
$$

and writing

$$
Q=\sqrt{x_{1} y_{0}}
$$

$$
y_{i}=y_{0} v_{i}
$$

one has to turn $\phi(\mathbf{v})$ in the previous computations into

$$
t\left(\frac{x_{0}}{x_{1}}+v_{1}+\frac{x_{2}}{x_{1}} v_{2}+\ldots+\frac{x_{n}}{x_{1}} v_{n}\right)-\frac{y_{0}}{q}\left(c_{1} v_{1}+\ldots+c_{n} v_{n}\right) .
$$


This slight modification, however, implies no significant modification in the proofs of Lemmas 9,10 and 12. Hence Proposition 8 remains valid if $\left(x_{0}, y_{0}\right)$ is replaced by $\left(x_{1}, y_{0}\right)$.

Recalling Theorem 1, it makes sense to speak about such expressions as error terms.

\section{On the main term}

Proposition 8 suggests that the main contribution to $N(B)$ comes from the summands with $\mathbf{c}=\mathbf{0}$. One is therefore led to evaluate

$$
\sum_{x_{0} y_{0}} w\left(x_{0}, y_{0}\right) \sum_{\mathbf{x}} w\left(x_{0}, \mathbf{x}\right) \sum_{q \ll \sqrt{x_{0} y_{0}}} q^{-n} S_{q}\left(x_{0}, y_{0}, \mathbf{x}\right)(\mathbf{0}) I_{q}\left(x_{0}, y_{0}, \mathbf{x}\right)(\mathbf{0}) .
$$

A necessary condition for

$$
S_{q}\left(x_{0}, y_{0}, \mathbf{x}\right)(\mathbf{0})=\sum_{d(q)}^{*} \sum_{\mathbf{b}(q)} e_{q}\left(d F\left(x_{0}, y_{0}, \mathbf{x}, \mathbf{b}\right)\right)
$$

to be different from zero is

$$
\mathbf{x} \equiv \mathbf{0}(q) .
$$

If $q>x_{0}$ this implies that $\mathbf{x}=\mathbf{0}$. However, as the origin is not contained in $\operatorname{supp} w\left(x_{0}, \mathbf{x}\right)$, this possibility may be excluded, and one may assume that $q \leqslant x_{0}$. Expression (6) can now be rewritten as

where

$$
\sum_{x_{0}, y_{0}} \frac{c\left(x_{0}, y_{0}\right)}{x_{0} y_{0}} w\left(x_{0}, y_{0}\right) \sum_{q \leqslant x_{0}} \sum_{\mathbf{a}(q)} q^{-n} S_{q}\left(x_{0}, y_{0}, \mathbf{a}\right)(\mathbf{0}) I\left(x_{0}, y_{0}, \mathbf{x}\right),
$$

$$
I\left(x_{0}, y_{0}, \mathbf{x}\right)=\sum_{\mathbf{x} \equiv \mathbf{a}(q)} w\left(x_{0}, \mathbf{x}\right) \int_{\mathbb{R}^{n}} w\left(y_{0}, \mathbf{y}\right) h\left(\frac{q}{\sqrt{\left|x_{0} y_{0}\right|}}, 1+\frac{\mathbf{x}}{x_{0}} \cdot \frac{\mathbf{y}}{y_{0}}\right) d \mathbf{y} .
$$

REMARK 15 . In case B a necessary condition for $S_{q}\left(x_{0}, y_{0}, \mathbf{x}\right)(\mathbf{0})$ to be different from zero is $x_{\max } \equiv 0(q)$. Thus, one may assume that $q \leqslant x_{\max }$.

Now, to ensure that condition (1) may be applied, a partition of the domain of integration is needed. One defines the weights

$$
w_{i}\left(x_{0}, \mathbf{x}\right)=w\left(x_{0}, \mathbf{x}\right)\left(1-\underline{\omega}_{\varepsilon}\left(1 / n-\left|x_{i}\right| / x_{0}\right)\right) \prod_{j=1}^{i-1} \underline{\omega}_{\varepsilon}\left(1 / n-\left|x_{j}\right| / x_{0}\right),
$$

for $i=1, \ldots, n-1$, and

Then one has

$$
w_{n}\left(x_{0}, \mathbf{x}\right)=\prod_{j=1}^{n-1} \underline{\omega}_{\varepsilon}\left(1 / n-\left|x_{j}\right| / x_{0}\right)
$$

$$
w\left(x_{0}, \mathbf{x}\right)=\sum_{j=1}^{n} w_{j}\left(x_{0}, \mathbf{x}\right),
$$

and $I\left(x_{0}, y_{0}, \mathbf{x}\right)$ can be written as the sum of the

$$
I_{i}\left(x_{0}, y_{0}, \mathbf{x}\right)=\sum_{\mathbf{x} \equiv \mathbf{a}(q)} w_{i}\left(x_{0}, \mathbf{x}\right) \int_{\mathbb{R}^{n}} w\left(y_{0}, \mathbf{y}\right) h\left(\frac{q}{\sqrt{\left|x_{0} y_{0}\right|}}, 1+\frac{\mathbf{x}}{x_{0}} \cdot \frac{\mathbf{y}}{y_{0}}\right) d \mathbf{y} .
$$


Replace now $y_{i}$ by

$$
t=1+\frac{\mathbf{x}}{x_{0}} \cdot \frac{\mathbf{y}}{y_{0}}
$$

to obtain

$$
I_{i}\left(x_{0}, y_{0}, \mathbf{x}\right)=x_{0} y_{0} \int_{\mathbb{R}} J_{i}(t) h\left(\frac{q}{\sqrt{x_{0} y_{0}}}, t\right) d t
$$

where

$$
J_{i}(t)=\sum_{\mathbf{x} \equiv \mathbf{a}(q)} \frac{w_{i}\left(x_{0}, \mathbf{x}\right)}{\left|x_{i}\right|} \int_{\mathbb{R}^{n-1}} w_{i}\left(y_{0}, t, \hat{\mathbf{y}}\right) d \hat{\mathbf{y}} .
$$

Here the 'hat' stands for the fact that the ith variable has been dropped, and $w_{i}\left(y_{0}, t, \hat{\mathbf{y}}\right)$ denotes the corresponding transformed weight function.

Next, one wants to make use of the fact that, by [9, Lemma 9], for small values of $x$ the function $h(x, y)$ acts very much like a delta-function. Precisely, one has the following lemma.

LEMma 16. Let $f$ be an infinitely differentiable function with compact support. Suppose that on $\operatorname{supp} f$

$$
\left|f^{(k)}(t)\right| \ll 1, \quad k=0,1,2, \ldots
$$

Then, for $s \ll 1$, and for any $N>0$

$$
\int f(t) h(s, t) d y=f(0)+O_{N}\left(s^{N}\right) .
$$

Condition (1) ensures that $w\left(y_{0}, t, \hat{\mathbf{y}}\right)$ and, as a consequence, $J_{i}(t)$ and the corresponding higher derivatives may be bounded by constants that depend only on $\varepsilon$ on $\operatorname{supp} w_{i}\left(x_{0}, \mathbf{x}\right) w\left(y_{0}, t, \hat{\mathbf{y}}\right)$. Hence, the hypotheses of Lemma 16 are fulfilled, and one concludes that $I_{i}\left(x_{0}, y_{0}, \mathbf{x}\right)$ can be written as

$$
x_{0} y_{0}\left(\sum_{\mathbf{x} \equiv \mathbf{a}(q)} \frac{w_{i}\left(x_{0}, \mathbf{x}\right)}{\left|x_{i}\right|} \int_{\mathbb{R}^{n-1}} w\left(y_{0}, 0, \hat{\mathbf{y}}\right) d \hat{\mathbf{y}}+O_{N}\left(\left(\frac{q}{\sqrt{x_{0} y_{0}}}\right)^{N}\right)\right) .
$$

Note, that by (2) the second term of (8) is $O_{N}(1)$. Its contribution to (7) is therefore bounded up to a multiplicative constant by

$$
\sum_{x_{0}, y_{0}} w\left(x_{0}, y_{0}\right) \sum_{1 \leqslant q \leqslant x_{0}} \sum_{\mathbf{a}(q)} q^{-n} S_{q}\left(x_{0}, y_{0}, \mathbf{a}\right)(\mathbf{0})\left(x_{0} / q\right)^{n} .
$$

Here one uses that $\left(x_{0} / q\right)^{n} \geqslant 1$. The fact that this expression is $O_{N}\left(B^{n / 2+1}\right)$, and therefore belongs to the error term, follows with $\beta=1$ from Lemma 17. This lemma will also be used later in the paper.

Lemma 17. For all $x_{0}, y_{0}$, and $\beta \geqslant 1$, one has

$$
\sum_{\beta \leqslant q} q^{-2 n} \sum_{\mathbf{a}(q)} S_{q}\left(x_{0}, y_{0}, \mathbf{a}\right)(\mathbf{0})=O\left(\beta^{-n+1}\right) .
$$

In case $B$ a similar result holds, except for $n=3$, and $x_{0} \equiv y_{0} \equiv 0(q)$. In that case one has

$$
\sum_{\beta \leqslant q} q^{-2 n-1} \sum_{\mathbf{a}(q)} S_{q}\left(x_{0}, y_{0}, \mathbf{a}\right)(\mathbf{0})=O\left(\beta^{-n+1}\right) .
$$


The first statement of Lemma 17 is a direct consequence of [9, Lemma 25] applied to the quadratic form $\mathbf{x} \cdot \mathbf{y}$.

The second statement follows from [9, Lemma 25] in a similar elementary way.

Note that the first statement is also a direct consequence of the precedent observation that

$$
S_{q}\left(x_{0}, y_{0}, \mathbf{a}\right)(\mathbf{0})= \begin{cases}q^{n} & \text { if } \mathbf{a} \equiv \mathbf{0}, \\ 0 & \text { if } \mathbf{a} \neq \mathbf{0} .\end{cases}
$$

REMARK 18. The treatment of the main term in case B is simpler, as no partition of the domain of integration is needed. If, say,

then replace $y_{1}$ by

$$
x_{1}=\max _{i}\left|x_{i}\right|, \quad y_{0}=\max _{i}\left|y_{i}\right|
$$

$$
t=\frac{x_{0}}{x_{1}}+\frac{y_{1}}{y_{0}}+\frac{x_{2} y_{2}}{x_{1} y_{0}}+\ldots+\frac{x_{n} y_{n}}{x_{1} y_{0}},
$$

and proceed as in case A. Note, that for $n=3$ one has to replace $\left(x_{0} / q\right)^{n}$ by $\left(x_{0} / q\right)^{n+1}$ in (9). The upper bound for (9) will be slightly weaker, but still sufficient.

Next, one wants to replace the sums over the $x_{i}$ by integrals. As $\varepsilon \leqslant 1 / 4 n$ one has

$$
\left(1-\underline{\omega}_{\varepsilon}\left(1 / n-\left|x_{i}\right| / x_{0}\right)\right) \underline{\omega}_{\varepsilon}\left(1 / 2 n-\left|x_{i}\right| / x_{0}\right)=0 .
$$

Thus, for $i=1, \ldots, n-1$, the weight $w_{i}\left(x_{0}, \mathbf{x}\right)$ can be written as

$$
\prod_{j=1}^{n} \tilde{w}_{j}\left(x_{0}, x_{j}\right)
$$

with factors that depend on $i$. For $i=n$ the weight may be written as a difference of two such products, and the subsequent argument is similar.

Write

$$
\sum_{\mathbf{x} \equiv \mathbf{a}(q)} \frac{w_{i}\left(x_{0}, \mathbf{x}\right)}{\left|x_{i}\right|}=\sum_{\mathbf{x} \equiv \mathbf{a}(q)} \frac{\tilde{w}_{i}\left(x_{0}, x_{i}\right)}{\left|x_{i}\right|} \prod_{j \neq i} \tilde{w}_{j}\left(x_{0}, x_{j}\right)
$$

or

$$
\sum_{\mathbf{z}} \prod_{j=1}^{n} \frac{\tilde{w}_{j}\left(x_{0}, a_{j}(q)+q z_{j}\right)}{\left|a_{i}(q)+q z_{i}\right|}
$$

For

$$
\sum_{z_{j}} \tilde{w}_{j}\left(x_{0}, a_{j}(q)+q z_{j}\right), \quad j \neq i,
$$

Euler's summation formula yields

$$
\int_{\mathbb{R}} \tilde{w}_{j}\left(x_{0}, a_{j}(q)+q z_{j}\right) d z_{j}+\int_{\mathbb{R}}\left(z_{j}-\left[z_{j}\right]\right) \frac{\partial \tilde{w}_{j}}{\partial z_{j}}\left(x_{0}, a_{j}(q)+q z_{j}\right) d z_{j} .
$$

Since $\partial \tilde{w}_{j} / \partial z_{j}$ is $O_{\varepsilon}\left(q x_{0}^{-1}\right)$, and is non-vanishing on a set of measure $O_{\varepsilon}\left(x_{0} q^{-1}\right)$, the last expression is

$$
\frac{1}{q} \int_{\mathbb{R}} \tilde{w}_{j}\left(x_{0}, x_{j}\right) d x_{j}+O_{\varepsilon}(1) .
$$


Similarly

can be evaluated by

$$
\sum_{z_{i}} \frac{\tilde{w}_{i}\left(x_{0}, a_{j}(q)+q z_{j}\right)}{\left|a_{i}(q)+q z_{i}\right|}
$$

$$
\frac{1}{q} \int_{\mathbb{R}} \frac{\tilde{w}_{i}\left(x_{0}, x_{i}\right)}{\left|x_{i}\right|} d x_{j}+O_{\varepsilon}\left(x_{0}^{-1}\right)
$$

Using the facts that

and

$$
\int_{\mathbb{R}} \tilde{w}_{j}\left(x_{0}, x_{j}\right) d x_{j}=O_{\varepsilon}\left(x_{0}\right)
$$

$$
\int_{\mathbb{R}} \frac{\tilde{w}_{i}\left(x_{0}, x_{i}\right)}{\left|x_{i}\right|} d x_{j}=O_{\varepsilon}(1),
$$

expression (10) may be replaced by

$$
q^{-n} \int_{\mathbb{R}^{n}} \frac{w_{i}\left(x_{0}, \mathbf{x}\right)}{\left|x_{i}\right|} d \mathbf{x}+O_{\varepsilon}\left(x_{0}^{n-2} q^{-n+1}\right) .
$$

Observe that the second summand belongs to an error term. Indeed, plugging this summand into (7) and treating the cases $q \leqslant \sqrt{x_{0}}$ and $q \geqslant \sqrt{x_{0}}$ separately, one is led on the one hand to evaluate after multiplication with an extra $x_{0}^{1 / 2} q^{-1}$-factor

$$
\sum_{x_{0}, y_{0}} w\left(x_{0}, y_{0}\right) x_{0}^{n-3 / 2} y_{0}^{n-1} \sum_{q \leqslant \sqrt{x_{0}}} \sum_{\mathbf{a}(q)} q^{-2 n} S_{q}\left(x_{0}, y_{0}, \mathbf{a}\right)(\mathbf{0}),
$$

where one makes use of

$$
\int_{\mathbb{R}^{n-1}} w\left(y_{0}, 0, \hat{\mathbf{y}}\right) d \hat{\mathbf{y}}=O_{\varepsilon}\left(y_{0}^{n-1}\right) .
$$

Lemma 17 then ensures that this expression is $O_{\varepsilon}\left(B^{n}\right)$. On the other hand one has to evaluate after multiplication with an extra $x_{0} q^{-1}$-factor

$$
\sum_{x_{0}, y_{0}} w\left(x_{0}, y_{0}\right) x_{0}^{n-1} y_{0}^{n-1} \sum_{\sqrt{x_{0}} \leqslant q \leqslant x_{0} \mathbf{a}(q)} q^{-2 n} S_{q}\left(x_{0}, y_{0}, \mathbf{a}\right)(\mathbf{0}) .
$$

By Lemma 17 this expression is bounded by

$$
\sum_{x_{0}, y_{0}} w\left(x_{0}, y_{0}\right) x_{0}^{n / 2-1 / 2} y_{0}^{n-1}=O_{\varepsilon}\left(B^{n}\right)
$$

REMARK 19. To be able to proceed in a similar way for $n=3$ in case $\mathrm{B}$, one has to split the summation in a slightly different way: $q \leqslant x_{0}^{1 / 3}, x_{0}^{1 / 3} \leqslant q \leqslant x_{0}^{2 / 3}, x_{0}^{2 / 3} \leqslant$ $q \leqslant x_{0}$.

Concerning the main term one is left to evaluate, after the transformations $\mathbf{x}=x_{0} \mathbf{u}$ and $\hat{\mathbf{y}}=y_{0} \hat{\mathbf{v}}$, the expressions

$$
\sum_{x_{0}, y_{0}} c\left(x_{0}, y_{0}\right) w\left(x_{0}, y_{0}\right) x_{0}^{n-1} y_{0}^{n-1} \sum_{q \leqslant x_{0}} \sum_{\mathbf{a}(q)} q^{-2 n} S_{q}\left(x_{0}, y_{0}, \mathbf{a}\right)(\mathbf{0}) \int_{\mathbb{R}^{2 n-1} \mid} \frac{1}{\left|u_{i}\right|} w_{i}(\mathbf{u}) w(\hat{\mathbf{v}}) d \hat{\mathbf{v}} d \mathbf{u},
$$

where $w_{i}(\mathbf{u})$ and $w(\hat{\mathbf{v}})$ denote the corresponding transformed weights. 
Since

$$
\sum_{x_{0}, y_{0}} c\left(x_{0}, y_{0}\right) w\left(x_{0}, y_{0}\right) x_{0}^{n-1} y_{0}^{n-1} \sum_{q \leqslant x_{0}} \sum_{\mathbf{a}(q)} q^{-2 n} S_{q}\left(x_{0}, y_{0}, \mathbf{a}\right)(\mathbf{0})
$$

is by Lemma 17 bounded up to a multiplicative constant by

$$
\sum_{x_{0}, y_{0}} c\left(x_{0}, y_{0}\right) w\left(x_{0}, y_{0}\right) y_{0}^{n-1},
$$

which is $O\left(B^{n}\right)$, one may sum in (11) over all $q \leqslant \sqrt{B}$. By the same argument one may reduce the summation to $q \leqslant \log (B)^{1 / 2-\varepsilon}$.

REMARK 20. To make this argument work in case B and for $n=3$ one has, as for previous contributions to the error term, to introduce an extra $x_{0} / q$-term.

Note at this stage that the integral in (11) contributes to the Tamagawa number at infinity. To see the link with the treatment of the constant in [9], one has to make use of [9, Theorem 3]. A 'light' version of this theorem is reproduced next.

THEOREM 21. Let $G\left(s_{1}, \ldots, s_{n}\right)$ be a real function, and $w\left(s_{1}, \ldots, s_{n}\right)$ be a smooth weight function. Suppose that the gradient of $G$ does not vanish on the closure of the support of $w$. Suppose that on the same set firstly $s_{i}$ is uniquely determined by the condition $G(s)=\mathbf{0}$, and secondly $\partial G / \partial s_{i}$ is positive and bounded from below. Then

$$
\lim _{\delta \rightarrow 0} \frac{1}{2 \delta} \int_{|G(\mathbf{s})| \leqslant \delta} w(\mathbf{s}) d \mathbf{s}=\int_{\mathbb{R}^{n-1}}\left(\frac{\partial G}{\partial s_{i}}\right)^{-1} w(\hat{\mathbf{s}}) d \hat{\mathbf{s}},
$$

where $s_{i}$ is given by $G(\mathbf{s})=\mathbf{0}$.

In the present case set

$$
G(\mathbf{u}, \mathbf{v})= \pm(1+\mathbf{u} \cdot \mathbf{v})
$$

depending on $\pm u_{i}>0$. As

$$
\frac{\partial G}{\partial v_{i}}= \pm u_{i}= \pm x_{i} / x_{0} \gg 1
$$

the hypotheses of Theorem 21 are satisfied on the two disjunct subsets of $\operatorname{supp} w_{i}(\mathbf{u}) w(\mathbf{v})$ characterised by $\pm u_{i}>0$. Hence the inner integral in the above expression may be replaced by

$$
\frac{1}{2 \delta} \int_{|1+\mathbf{u} \cdot \mathbf{v}| \leqslant \delta} w_{i}(\mathbf{u}) w(\mathbf{v}) d \mathbf{v} d \mathbf{u}+O(\delta),
$$

for $\delta$ arbitrarily small.

Let $w(\mathbf{u})$ be the sum of the $w_{i}(\mathbf{u})$. Set

Remark that

$$
\sigma_{(\mathbf{0}, 0)}(w)=\lim _{\delta \rightarrow 0} \frac{1}{2 \delta} \int_{|1+\mathbf{u} \cdot \mathbf{v}| \leqslant \delta} w(\mathbf{u}) w(\mathbf{v}) d \mathbf{u} d \mathbf{v} .
$$

$$
\sigma_{(0,0)}(w)=\hat{\sigma}_{(0,0)}+O(\varepsilon),
$$

with $\hat{\sigma}_{(0,0)}=\sigma_{(0,0)}(\chi)$, where $\chi$ is the characteristic function of the 1-box at the origin.

REMARK 22. In a similar way one obtains constants $\hat{\sigma}_{(i, j)}$ for the remaining pairs $(i, j)$. 
As the third sum in

is

$$
\sum_{q \leqslant \log (B)^{1 / 2-\varepsilon}} \sum_{\substack{a_{0}(q), a_{0}^{\prime}(q) \\ \mathbf{a}(q)}} q^{-2 n} S_{q}\left(a_{0}, a_{0}^{\prime}, \mathbf{a}\right)(\mathbf{0}) \sum_{\substack{x_{0}=a_{0}(q) \\ y_{0} \equiv a_{0}^{\prime}(q)}} c\left(x_{0}, y_{0}\right) w\left(x_{0}, y_{0}\right) x_{0}^{n-1} y_{0}^{n-1}
$$

$$
\frac{1}{q^{2}}\left(\frac{1}{2 n} B^{n} \log (B)+O_{\varepsilon}\left(B^{n} \log (B)^{1-2 \varepsilon}\right)\right),
$$

it remains to evaluate

where

$$
\sum_{q \leqslant \log (B)^{1 / 2-\varepsilon}} q^{-(2 n+2)} S_{q}(0)
$$

$$
S_{q}(0)=\sum_{\mathbf{a}(q), a_{0}(q), a_{0}^{\prime}(q)} S_{q}(\mathbf{a})(\mathbf{0}) .
$$

Following the final part of [9, Section 11], one begins with the observation that the corresponding infinite sum is absolutely convergent, and that one can show that

$$
\sum_{q \leqslant \log (B)^{1 / 2-\varepsilon}} q^{-(2 n+2)} S_{q}(\mathbf{0})=\sum_{q=1}^{\infty} q^{-(2 n+2)} S_{q}(\mathbf{0})+O\left(\log (B)^{(1-n) / 2+\varepsilon}\right) .
$$

Moreover $q^{-(2 n+2)} S_{q}(\mathbf{0})$ is multiplicative. Therefore

where

$$
\sum_{q=1}^{\infty} q^{-(2 n+2)} S_{q}(\mathbf{0})=\prod_{p \text { prime }} \sigma_{p},
$$

$$
\sigma_{p}=\sum_{t=0}^{\infty} p^{-(2 n+2) t} S_{p^{t}}(\mathbf{0})
$$

or, as in the usual analysis of the singular series,

$$
\sigma_{p}=\lim _{k \rightarrow \infty} p^{-(2 n+1) k} \operatorname{card}\left\{(\mathbf{x}, \mathbf{y})\left(p^{k}\right) \mid \mathbf{x} \times \mathbf{y} \equiv 0\left(p^{k}\right)\right\} .
$$

The various findings can be summarised as follows.

Proposition 23. One has

$$
\sum_{i, j} N\left(F, w_{\varepsilon, B,(i, j)}\right)=\frac{1}{2 n} \prod_{p} \sigma_{p}\left(\sum_{i, j} \hat{\sigma}_{(i, j)}\right) B^{n} \log (B)(1+o(1)),
$$

and in particular

$$
\sum_{i, j} N\left(F, \underline{w}_{\varepsilon, B,(i, j)}\right) \sim \sum_{i, j} N\left(F, \bar{w}_{\varepsilon, B,(i, j)}\right) .
$$

The following corollary is an immediate consequence.

Corollary 24. One has

$$
N(B)=\frac{1}{n} \prod_{p} \sigma_{p} \sigma_{\infty} B^{n} \log (B)(1+o(1)),
$$

where

$$
\sigma_{\infty}=\sum_{i, j} \hat{\sigma}_{(i, j)} .
$$




\section{On the constant}

To derive Theorem 1 from Corollary 24 one writes

$$
N(X, \phi, B)=\sum_{l}^{\infty} \sum_{k}^{\infty} \mu(l) \mu(k) N(B / k l),
$$

where $\mu$ denotes the standard Möbius function. Therefore

where

$$
N(X, \phi, B)=\frac{1}{n} \prod_{p} \sigma_{p}^{\prime} \sigma_{p} \sigma_{\infty} B^{n} \log (B)(1+o(1)),
$$

$$
\sigma_{p}^{\prime}=\left(1-p^{-n}\right)^{2} .
$$

This concludes the proof of Theorem 1. Finally some considerations should be made on the constant. Observe that

$$
\sigma_{p}^{\prime} \sigma_{p}=\lim _{k \rightarrow \infty} p^{-(2 n+1) k} \operatorname{card}\left\{(\mathbf{x}, \mathbf{y})\left(p^{k}\right) \mid \mathbf{x} \times \mathbf{y} \equiv 0\left(p^{k}\right),(\mathbf{x}, p)=(\mathbf{y}, p)=1\right\} .
$$

The model has good reduction. Hence the limit stabilises at $k=1$, and

$$
\sigma_{p}^{\prime} \sigma_{p}=\frac{\operatorname{card}\left\{\mathscr{T}\left(\mathbb{F}_{p}\right)\right\}}{p^{2 n+1}}=\frac{\operatorname{card}\left\{\mathbb{G}_{m}^{2}\left(\mathbb{F}_{p}\right)\right\}}{p^{2}} \frac{\operatorname{card}\left\{\tilde{Y}\left(\mathbb{F}_{p}\right)\right\}}{p^{2 n-1}},
$$

with $\mathbb{F}_{p}=\mathbb{Z} / p \mathbb{Z}$.

As $\sigma_{\infty}$ is the Tamagawa factor at infinity, $\sigma_{\infty} \prod_{p} \sigma_{p}^{\prime} \sigma_{p}$ admits an interpretation as the Tamagawa number of the variety $X$, which is in accordance with $[\mathbf{1 2}, \mathbf{1 6}]$.

In [12] it is conjectured that, beside the Tamagawa number of the variety, an additional constant should appear in the asymptotical formula. It should be equal to a particular measure of a domain in $\mathbb{R}^{\rho}$, where $\rho$ is the rank of Pic $X$. For a precise statement see [12, Definition 2.4].

In the present case the 1-dimensional domain in $\mathbb{R}^{2}$ that has to be considered is given with respect to the anticanonical sheaf by

$$
z_{1}, z_{2} \geqslant 0, \quad n z_{1}+n z_{2}=1
$$

and the constant turns out to be equal to $1 / n^{2}$.

Passing from $O(n, n)$ to $O(1,1)$ involves a factor $n$. Therefore the present result is in accordance with Peyre's conjecture, and the asymptotical formula is in accordance with the general philosophy on the interplay of geometry and arithmetics on Fano varieties.

Acknowledgements. The author would like to express his gratitude to D. R. Heath-Brown, for the invitation to Oxford, for many helpful discussions, and for his patience in reading an earlier, far from flawless draft of this paper.

The author is also indebted to P. Salberger, for having suggested the problem and [9] as a key to its solution, and to D. Coray, for his constant interest in this work.

It is also a pleasure to record the logistical support of the Mathematical Institute, Oxford, where this work was carried out, and the financial support of the Swiss National Research Foundation, which was a conditio sine qua non for this research. 


\section{References}

1. V. Batyrev and Y. Manin, 'Sur le nombre de points rationnels de hauteur bornée des variétés algébriques', Math. Ann. 286 (1990) 27-43.

2. V. BATYREV and Y. TSCHINKel, 'Rational points of bounded height on compactifications of anisotropic tori', Internat. Math. Res. Notices 12 (1995) 591-635.

3. V. BAtYrev and Y. Tschinkel, 'Manin's conjecture for toric varieties', J. Algebraic Geom. 7 (1998) $15-53$.

4. B. J. BirCH, 'Forms in many variables', Proc. Roy. Soc. London 265A (1962) 245-263.

5. W. Duke, J. Friedlander and H. IwanieC, 'Bounds for automorphic $L$-functions', Invent. Math. 112 (1993) $1-8$.

6. J. Franke, Y. Manin and Y. Tschinkel, 'Rational points of bounded height on Fano varieties', Invent. Math. 95 (1989) 421-435.

7. R. Hartshorne, Ample subvarieties of algebraic varieties, Lecture Notes in Mathematics 156 (Springer, 1970).

8. D. R. Heath-Brown, 'Cubic forms in ten variables', Proc. London Math. Soc. (3) 47 (1983) 225-257.

9. D. R. Heath-Brown, 'A new form of the circle method, and its application to quadratic forms', J. Reine Angew. Math. 481 (1996) 149-206.

10. S. LANG, Fundamentals of Diophantine geometry (Springer, 1983).

11. S. LANG (ed.), 'Number theory III', Encyclopaedia of mathematical sciences - Vol. 60 (Springer, 1991).

12. E. Peyre, 'Hauteurs et mesures de Tamagawa sur les variétés de Fano', Duke Math. J. 79 (1995) $101-218$.

13. E. Peyre, 'Terme principal de la fonction zeta des hauteurs et torseurs universels', Astérisque 251 (1998) 259-298.

14. E. Peyre, 'Torseurs universels et méthode du cercle', preprint, 1998.

15. M. Robbiani, 'On the arithmetic of isotropic Del Pezzo surfaces of degree six', J. Reine Angew. Math. 503 (1998) 1-45.

16. P. Salberger, 'Tamagawa measures on universal torsors and points of bounded height on Fano varieties', Astérisque 251 (1998) 91-258.

17. S. Schanuel, 'Heights in number fields', Bull. Soc. Math. France 107 (1979) 433-449.

18. W. Sснмidt, 'The density of integer points on homogeneous varieties', Acta Math. 154 (1985) $243-296$.

19. E. Stein, Harmonic analysis, Princeton Mathematical Series 43 (Princeton University Press, 1993).

20. J. L. Thunder, 'Asymptotic estimates for rational points of bounded height on flag varieties', Comp. Math. 88 (1993) 155-186.

ETH Zürich

Mathematik

CH-8092 Zürich

Switzerland

robbiani@math.ethz.ch

rob@zhwin.ch 